Math. Model. Nat. Phenom.

Vol. 5, No. 6, 2010, pp. 96-108

DOI: $10.1051 / \mathrm{mmnp} / 20105605$

\title{
A New Mathematical Model of Syphilis
}

\author{
F. A. Milner $^{1 *}$ and R. Zhao ${ }^{2}$ \\ ${ }^{1}$ School of Mathematical and Statistical Sciences, Arizona State University \\ P.O. Box 871804, Tempe, AZ 85287-1804, USA \\ ${ }^{2}$ Department of Computer Science, Purdue University, West Lafayette, IN 47907-2107, USA
}

\begin{abstract}
The CDC launched the National Plan to Eliminate Syphilis from the USA in October 1999 [4]. In order to reach this goal, a good understanding of the transmission dynamics of the disease is necessary. Based on a SIRS model Breban et al. [3] provided some evidence that supports the feasibility of the plan proving that no recurring outbreaks should occur for syphilis. We study in this work a syphilis model that includes partial immunity and vaccination. This model suggests that a backward bifurcation very likely occurs for the real-life estimated epidemiological parameters for syphilis. This may explain the resurgence of syphilis after mass treatment [21]. Occurrence of backward bifurcation brings a new challenge for the plan of the CDC's -striking a balance between treatment of early infection, vaccination development and health education. Our models suggest that the development of an effective vaccine, as well as health education that leads to enhanced biological and behavioral protection against infection in high-risk populations, are among the best ways to achieve the goal of elimination of syphilis in the USA.
\end{abstract}

Key words: backward bifurcation, partial immunity, vaccination, syphilis AMS subject classification: $92 \mathrm{D} 30$

\section{Introduction}

Sir William Osler said "Syphilis is a great imitator." [20] In fact, it was only identified as a distinct infection from gonorrhea by Phillippe Ricord in 1838. Syphilis is a human infectious disease, transmitted almost always through sexual contact, caused by the spirochete Treponema pallidum

*Corresponding author. E-mail: milner@asu.edu 
subspecies pallidum, and first recognized in Western Europe following Columbus' return from the Americas in 1500. T. pallidum subspecies pallidum belongs to the family of Spirochaetaceae (spirochetes), or spiral-shaped bacteria, and is related to other pathogenic treponemes that cause nonvenereal diseases, e.g. T. pallidum subspecies endemicum (bejel), T. pallidum subspecies pertenue (yaws), and T.carateum (pinta), which can be differentiated from T.pallidum subspecies pallidum by the clinical manifestations of their respective diseases as well as by genetic differences. T. pallidum varies from 6 to $15 \mu \mathrm{m}$ in length and is $0.2 \mu \mathrm{m}$ in diameter.

Syphilis is a multistage disease that progresses, when untreated, from primary to secondary and, finally, to tertiary infection. Partial immunity is acquired in both treated and untreated syphilis. It is a disease of a considerable public health importance because, if not treated, it can lead to various cardiovascular and neurological diseases as well as to adverse pregnancy outcomes, such as stillbirth and congenital syphilis [6]. Also, it is estimated that $15 \%$ of blindness was due to syphilis in 1900, when the disease was still incurable [20]. Nowadays early syphilis can be easily treated by a single dose of antibiotics such as penicillin or azithromycin. The World Health Organization estimated that 12 million new cases of syphilis occur each year in the world and the vast majority of them affect people who live in developing countries [16].

Syphilis was believed to be near extinction in the USA and other developed countries due to widespread use of penicillin beginning around 1945. Incidence of syphilis in the USA during WWII was over 500,000 infections per year. Between 1945 and 2000 it declined to 31,575 reported infections per year, with cyclic peaks and troughs of infectious cases. However, resurgence of syphilis in several countries has been observed in several instances since the 1970s. These more recent outbreaks can be explained by many factors: the gay liberation movement of the 1970s [3], crack cocaine-related prostitution among women in the late 1980s [13, 19], changes in the intensity of the syphilis control program, as well as changes in sexual behavior, and the HIV epidemic in the 1990s [15, 21], or random fluctuations in composition of the population [6]. Examination of these outbreaks reveals a common thread: the presence of a "core group" of high-activity, high-risk individuals that drives and determines the dynamics of the epidemics.

Garnett et al. were the first to study syphilis using a mathematical model that include all stages of the disease described by Phillippe Ricord [8]. They assumed that infected individuals acquire temporary immunity only after recovery from the latent and tertiary infections. However, they did not focus on behavioral changes (e.g. protection during sexual contact through condom use [22]), which is an important component in the CDC's eradication plan. That plan has specified three types of intervention to reach the goal: enhancement of public health services; evidence-based interventions that are culturally appropriate; and accountability.

Behavioral protection against sexually transmitted diseases is important because it affects the dynamics of the disease in the population. Reluga et al. showed that, if resistance is behaviorally induced, backward bifurcations may occur when reinfection causes an elevated rate of loss of resistance. In contrast, backward bifurcations do not occur when resistance is immunologically induced [22]. Recently Grassly et al. fitted real life data to a SIRS model and estimated an epidemic period of 8-11 years [13]. However, Breban et al. found no evidence of cyclic recurrence studying the same model, seemingly providing support for the Syphilis Elimination Effort launched by the CDC to eradicate syphilis in the USA [3]. 
In this paper, we propose a new mathematical model based on the assumption that secondary and later infections confer partial immunity. We also consider two different sources of protection: inoculation and behavior. We shall refer to vaccination as biological protection to contrast it with behavioral protection resulting from changes in patterns of sexual contact. The susceptible population in the core group could, for example, adopt safe sex habits after an improvement in public health education.

At this time a vaccine for syphilis is still under development and it only confers partial immunity; however, at least one animal study has demonstrated complete protection against T. pallidum [16]. Therefore, we also include vaccination in our model as a possible tool for controlling syphilis. We also assume that partial immunity against reinfection ensures both from treatment and vaccination.

Our results suggest that the resurgence of syphilis in the last several decades could be the result of a backward bifurcation. One of the main goals of this paper is to provide a warning -a possible backward bifurcation for the transmission dynamics of syphilis- by studying three mathematical models and estimating the minimum effort necessary to avoid failure in the eradication effort.

In the next section we describe the SIRS model analyzed in [13]; in Section 3 we introduce and analyze our new model with a single phase of infection, and in Section 4 we introduce the model with multiple stages. Finally, in Section 5, we discuss our findings and draw some conclusions.

\section{A SIRS model of syphilis}

We begin by describing the deterministic SIRS model used by Grassly et al. [13]. The population under consideration is divided into susceptible, infected, and recovered individuals within a "core group" that is very sexually active and which is believed to cause the epidemic. The immunity acquired after treatment is temporary.

Let $S(t), I(t)$, and $R(t)$ be the fractions of susceptible, infected, and recovered individuals in the population at time $t$, respectively. The model is based on the assumption of demographic equilibrium and consists of the following system of ODEs:

$$
\begin{aligned}
& S^{\prime}=\mu-\beta I S-\mu S+\nu R \\
& I^{\prime}=\beta I S-\gamma I-\mu I \\
& R^{\prime}=\gamma I-\mu R-\nu R
\end{aligned}
$$

where $\beta$ is the transmission parameter that synthesizes both the contact rate and the virulence (i.e. probability of transmission per contact), $\mu$ is per capita rate of death/birth, $\nu$ is the rate of loss of immunity, and $\gamma$ is the natural recovery rate (i.e. $1 / \gamma$ is the mean infectious period for untreated syphilis).

The disease-free equilibrium (DFE) for the model $(2.1)$ is $\left(S_{1}^{*}, I_{1}^{*}, R_{1}^{*}\right)=(1,0,0)$, and the basic reproduction number,

$$
\mathcal{R}_{0,1}=\frac{\beta}{\mu+\gamma}
$$


represents "the expected number of secondary cases produced, in a completely susceptible population, by a typical infective individual" [5]. The disease-free equilibrium is locally asymptotically stable if the basic reproduction number is less than 1 .

Breban et al. lent support to the feasibility of the CDC's elimination plan by showing that no cycling occurs for this model [3]. However, mathematically speaking, eradicability of a disease depends on whether the disease-free equilibrium is globally stable or not. If a backward bifurcation occurs, the condition that the basic reproduction number is less than 1 is no longer enough to guarantee the ultimate elimination of the disease.

Following the method of Dushoff et al. [7], a backward bifurcation occurs for a compartmental model as the basic reproduction number equals to 1 if, and only if, $h=\mathbf{V}^{*} \cdot H^{\prime} \mathbf{V}^{0}>0$, where $\mathbf{V}^{0}$ and $\mathbf{V}^{*}$ are the right and left eigenvectors of $H(\hat{V})$ corresponding to the dominant eigenvalue 0 , and $H^{\prime}=d H\left(\hat{V}+\alpha V^{0}\right) /\left.d \alpha\right|_{\alpha=0}$, where $H$ rewrites the model as $d V / d t=H(V)(V-\hat{V})$, $V$ represents the dynamical variables and $\hat{V}$ is an equilibrium. For example, $V=(I, S, R)$, $\hat{V}=(0,1,0)$ in the model $(2.1)$.

We can see that a necessary and sufficient condition for the model (2.1) to have a backward bifurcation at $\mathcal{R}_{0,1}=1$ is

$$
\gamma \nu>\beta(\mu+\nu)
$$

Since Eq. (2.3) is obviously false for $\mathcal{R}_{0,1}=\frac{\beta}{\mu+\gamma}=1$, (2.2), there is no backward bifurcation for model (2.1).

\section{A new modified SIR model of syphilis}

The average primary incubation period of syphilis is about 25 days; primary infection lasts some 46 days before progression to secondary infection takes place; secondary infection lasts approximately 3.6 months and leads to the latent stage [8]. In both treated and untreated syphilis, partial immunity gradually develops due to acquired immune memory and continued presence of antigens respectively [17].

In the present paper we assume that infection with syphilis leads to partial immunity if it progresses to secondary or later stage infection. We study the epidemic using models with suboptimal immunity first proposed by Hadeler and Van den Driessche [14]. Gomes et. al. later applied these models to tuberculosis [9] and influenza [12] with an emphasis on reinfection [10,11].

We now introduce a simple mathematical model of syphilis with sub-optimal immunity but no stage of infection. Let $\sigma$ be the factor of reduction of susceptibility for individuals in the recovered class, let $v$ be the protection rate (both by vaccination and by safe sex activity), and let $p$ be the fraction of primary infections going back to the susceptible pool among all infected individuals. Then the dynamics of transmission can be described by the following special case of a model in [22]:

$$
\begin{aligned}
& S^{\prime}=\mu-\beta I S+p \gamma I-v S-\mu S, \\
& I^{\prime}=\beta I(S+\sigma R)-\gamma I-\mu I, \\
& R^{\prime}=(1-p) \gamma I-\sigma \beta I R+v S-\mu R .
\end{aligned}
$$

The parameters $\mu, \gamma$, and $\beta$ here have the same meaning as in (2.1). 
The disease-free equilibrium of the model (3.1) is

$$
\left(S_{2}^{*}, I_{2}^{*}, R_{2}^{*}\right)=\left(\frac{\mu}{\mu+v}, 0, \frac{v}{\mu+v}\right)
$$

and the basic reproduction number is

$$
\mathcal{R}_{0,2}=\left(\frac{\beta}{\mu+\gamma}\right)\left(\frac{\mu}{\mu+v}\right)+\sigma\left(\frac{\beta}{\mu+\gamma}\right)\left(\frac{v}{\mu+v}\right) .
$$

The basic reproduction number $\mathcal{R}_{0,2}$ consists of two terms, the first representing infections of susceptible individuals and the second infections of vaccinated ones. We see that $\mathcal{R}_{0,2}$ is a decreasing function of $v$, and an increasing function of $\beta$ and $\sigma$.

The argument of Dushoff et al. [7] leads us to conclude that a backward bifurcation for the model (3.1) occurs at $\mathcal{R}_{0,2}=1$ if, and only if,

$$
\gamma(1-\sigma)(\mu+\sigma v) p>\gamma \mu(1-\sigma)+\sigma^{2} v^{2}+\mu v \sigma(1+\sigma)+\mu^{2} .
$$
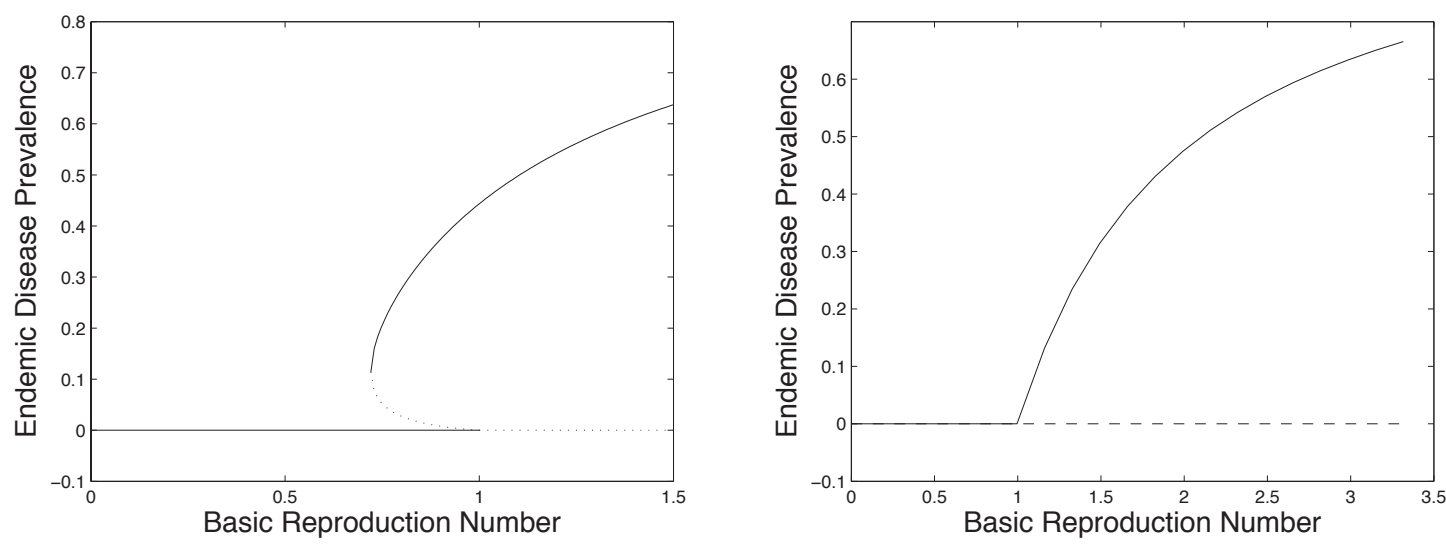

Figure 1: Bifurcation plots. Solid lines indicate asymptotically stable equilibria, dashed lines unstable. The graph on the left shows a backward bifurcation for (3.1) using $p=0.8, \sigma=0.3$ and $\beta$ varying so that $\mathcal{R}_{0,2}$ and $I$ change. The graph on the right shows a forward bifurcation for (2.1). The DFE for (2.1) is globally stable if $\mathcal{R}_{0,1}<1$ but (3.2) is only locally stable and there is another stable positive equilibrium when $R_{0,2}^{*}<\mathcal{R}_{0,2}<1$ for some $R_{0,2}^{*}$.

Figure 1 contrasts a forward bifurcation for the model (2.1) and a backward bifurcation for the model (3.1) above. Eq. (3.4) can be satisfied for realistic parameter values for syphilis. In particular, we take values from Breban et al. [2]: $\beta=9.045, \gamma=6, \mu=0.03, v$ and $\sigma$ are chosen so that $\mathcal{R}_{0,2}=1$, see (3.3).

Figure 2 shows the $p$ - $\sigma$ region in which a backward bifurcation occurs at $\mathcal{R}_{0,2}=1$. If we take the protection rate $v=0.3$, then $\sigma=0.6333$ makes $\mathcal{R}_{0,2}=1$. If $p>0.232$, then a backward 


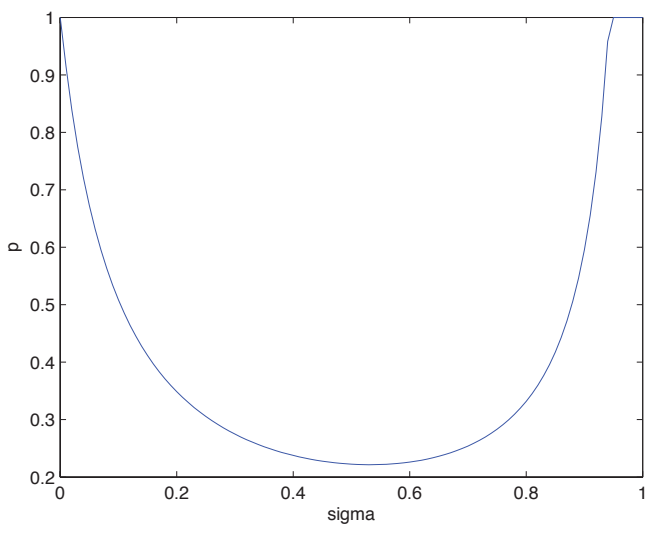

Figure 2: $p$ - $\sigma$ diagram for a backward bifurcation. A backward bifurcation occurs in the upper region in the graph represented by condition (3.4).

bifurcation occurs. If mass treatment is mainly aimed at primary infections, then $p$ will be much higher than 0.232 . In other words, it is very likely that a backward bifurcation occurs for syphilis if the protection efficacy is relatively low.

We assume that recovery from primary infection does not confer immunological protection against reinfection. This feature of immune response suggests that the treatment in earlier stages of syphilis increases the susceptible pool, thus making a backward bifurcation more likely and the eradication of the disease more difficult.

On the other hand, with mass treatment of primary syphilis such that $p>(\sigma+\mu(1+\sigma)) /(\gamma(1-$ $\sigma)$ ), a backward bifurcation occurs for (3.1) if

$$
v>\frac{\gamma \mu(1-\sigma)(1-p)+\mu^{2}}{\sigma[\gamma(1-\sigma) p-\sigma-\mu(1+\sigma)]}
$$

This means that, if the protection efficacy is not improved, the broader the coverage of protection is, the more likely a backward bifurcation becomes. Note that the basic reproduction number $\mathcal{R}_{0,2}$ is a decreasing function of $v$. The drawback to improving protection coverage is the same as that of treating individuals in early stages: a backward bifurcation becomes more likely.

Since a backward bifurcation is likely to occur for syphilis, any strategy of elimination of syphilis should not only aim at decreasing the basic reproduction number $\mathcal{R}_{0,2}$ below 1 , but also at ensuring that the disease-free equilibrium is a global attractor. In order to achieve this for model (3.1), a necessary condition that we shall assume henceforth is

$$
[\gamma+\mu-\sigma \beta-(1-\sigma) p \gamma+\sigma(\mu+v)]^{2}<4 \sigma[(\mu+v)(\gamma+\mu-\sigma \beta)-\beta(1-\sigma) \mu] .
$$

Behavioral and social factors can be interpreted in terms of epidemiological parameters in this model. Some effects can be viewed as included in $v$, such as the at-risk population starting to use protective measures (e.g. condom use as response to education and awareness); others 
can be included in $\sigma$ (e.g. individuals becoming more careful in selecting sexual partners after treatment because of increased awareness, thus decreasing the probability of transmission); the same explanation applies to $p$ since recovery from primary infection does not induce biological protection but, possibly, behavioral protection against reinfection.

\section{A new model of syphilis with multiple stages}

In order to have a better understanding of how the existence of different stages can affect control strategies, especially through the existence of backward bifurcations, it is necessary to study a more complete, albeit more complex, model that includes all stages of the disease. Just as in [8], we shall follow the natural history of syphilis: primary infection, secondary infection, latent infection, and tertiary infection. Longer and stronger treatment is needed for later stages of syphilis.

Let $S(t)$ denote the proportion of susceptible individuals; $I_{1}(t), I_{2}(t)$ denote, respectively, the proportions of individuals with primary infection from the susceptible pool and from the partially biologically immune class, respectively; $I_{s}(t), L(t), I_{t}(t)$ denote the proportions of individuals with secondary infection, latent infection, and tertiary infection, respectively; $R_{1}(t)$ denote the proportion of individuals recovered from $I_{1}$ and those from $S$ adopting safe sex habit; finally, $R_{2}$ denote the proportion of individuals recovered from $I_{2}, I_{s}, L$ and $I_{t}$ and those vaccinated in $S$;

We shall make the following assumptions: susceptible individuals who have contact with individuals with primary or secondary infection become infected at per capita rate $\beta$; if untreated, primary infection progresses to secondary infection at rate $\varphi_{1}$, then it progresses to latent infection at rate $\varphi_{2}$ and, finally, goes into tertiary infection at rate $\varphi_{3}$; recovered individuals either become fully susceptible, with probability $f$, or develop partial immunity (i.e. $1-f$ is the fraction of treated patients in $I_{1}$ who adopt behavioral protection against reinfection). Also, we shall assume that the protective effect of vaccines is indistinguishable from that of recovery with partial immunity -thus giving a clear meaning to "biologically induced immunity."

Let $v_{1}$ be the rate at which susceptible individuals develop safe sex habits as a consequence of health care education (i.e. the rate at which they develop "behavioral immunity") and $v_{2}$ be the rate of vaccination of the susceptible population (i.e. the rate at which they develop "biological immunity"); let $\sigma_{1}$ be the rate of reduction of reinfection due to behavioral protection, and $\sigma_{2}$ due to biological protection.

The transmission dynamics of syphilis is then described by the following system of ordinary differential equations, each describing how one of the classes evolves in time,

$$
\begin{aligned}
& S^{\prime}=\mu+f \gamma I_{1}-\beta\left(I_{1}+I_{2}+I_{s}\right) S-\left(v_{1}+v_{2}\right) S-\mu S, \\
& I_{1}^{\prime}=\beta\left(I_{1}+I_{2}+I_{s}\right)\left(S+\sigma_{1} R_{1}\right)-\left(\gamma+\varphi_{1}+\mu\right) I_{1}, \\
& R_{1}^{\prime}=(1-f) \gamma I_{1}+v_{1} S-\sigma_{1} \beta\left(I_{1}+I_{2}+I_{s}\right) R_{1}-\mu R_{1}, \\
& I_{2}^{\prime}=\sigma_{2} \beta\left(I_{1}+I_{2}+I_{s}\right) R_{2}-\left(\gamma+\varphi_{1}+\mu\right) I_{2}, \\
& I_{s}^{\prime}=\varphi_{1}\left(I_{1}+I_{2}\right)-\left(\gamma+\varphi_{2}+\mu\right) I_{s}, \\
& L^{\prime}=\varphi_{2} I_{s}-\left(\gamma+\varphi_{3}+\mu\right) L, \\
& I_{t}^{\prime}=\varphi_{3} L-(\gamma+\mu) I_{t}, \\
& R_{2}^{\prime}=\gamma\left(I_{2}+I_{s}+L+I_{t}\right)+v_{2} S-\sigma_{2} \beta\left(I_{1}+I_{2}+I_{s}\right) R_{2}-\mu R_{2} .
\end{aligned}
$$


Parameters appearing in (4.1) with the same name as in the models (2.1) and (3.1) have the same meaning as in those.

Once again we use the method of Dushoff et al. [7], described at the end of Section 2, to find conditions for a backward bifurcation for the model (4.1). We shall provide here a detailed derivation.

For convenience, we introduce some new notation:

$$
\begin{array}{lll}
\Pi_{0}=\frac{\beta}{\gamma+\mu+\varphi_{1}}, & \Pi_{0}^{*}=\frac{\sigma_{2} v_{2}}{\mu+\sigma_{1} v_{1}}, \\
\Pi_{1}=\frac{\varphi_{2}}{\gamma+\varphi_{2}+\mu}, & \Pi_{2}=\frac{\varphi_{2}}{\gamma+\varphi_{3}+\mu}, & \Pi_{3}=\frac{\varphi_{3}}{\gamma+\mu}, \\
S_{0}=\frac{v_{1}}{\mu+v_{1}+v_{2}}, & R_{10}=\frac{v_{2}}{\mu+v_{1}+v_{2}}, & R_{20}=\frac{v_{1}+v_{2}}{\mu+v_{1}} .
\end{array}
$$

Denote

$$
V=\left(I_{1}, I_{2}, I_{s}, L, I_{t}, S, R_{1}, R_{2}\right) .
$$

Then, the disease-free equilibrium of (4.1) is

$$
\hat{V}=\left(I_{10}^{*}, I_{20}^{*}, I_{s}^{*}, L^{*}, I_{t}^{*}, S^{*}, R_{10}^{*}, R_{20}^{*}\right)=\left(0,0,0,0,0, S_{0}, R_{10}, R_{20}\right)
$$

and, using the notation (4.2), the basic reproduction number (calculated as in [23])

$$
\mathcal{R}_{0,3}=\Pi_{0}\left(1+\Pi_{1}\right)\left(S_{0}+\sigma_{1} R_{10}+\sigma_{2} R_{20}\right),
$$

consisting of six terms - two each for new infections of susceptible individuals, of those with behavioral protection, and of those biologically protected by vaccination.

Clearly, $H$ introduced at the end of Section 2 has the following form:

$$
\left(\begin{array}{cccccccc}
h_{11} & \beta\left(S+\sigma_{1} R_{1}\right) & \beta\left(S+\sigma_{1} R_{1}\right) & 0 & 0 & 0 & 0 & 0 \\
\sigma_{2} \beta R_{2} & h_{22} & \sigma_{2} \beta R_{2} & 0 & 0 & 0 & 0 & 0 \\
\varphi_{1} & \varphi_{1} & h_{33} & 0 & 0 & 0 & 0 & 0 \\
0 & 0 & \varphi_{2} & h_{44} & 0 & 0 & 0 & 0 \\
0 & 0 & 0 & \varphi_{3} & h_{55} & 0 & 0 & 0 \\
-\beta S+f \gamma & -\beta S & -\beta S & 0 & 0 & h_{66} & 0 & 0 \\
-\sigma_{1} \beta R_{1}+(1-f) \gamma & -\sigma_{1} \beta R_{1} & -\sigma_{1} \beta R_{1} & 0 & 0 & v_{1} & -\mu & 0 \\
-\sigma_{2} \beta R_{2} & -\sigma_{2} \beta R_{2}+\gamma & -\sigma_{2} \beta R_{2}+\gamma & \gamma & \gamma & v_{2} & 0 & -\mu
\end{array}\right)
$$

where

$$
\begin{aligned}
& h_{11}=\beta\left(S+\sigma_{1} R_{1}\right)-\left(\gamma+\varphi_{1}+\mu\right), \\
& h_{22}=\sigma_{2} \beta R_{2}-\left(\gamma+\varphi_{1}+\mu\right), \\
& h_{33}=-\left(\gamma+\varphi_{2}+\mu\right), \\
& h_{44}=-\left(\gamma+\varphi_{3}+\mu\right), \\
& h_{55}=-(\gamma+\mu), \\
& h_{66}=-\left(\mu+v_{1}+v_{2}\right) .
\end{aligned}
$$


Moreover, as introduced at the end of Section 2, here we have

$$
\mathbf{V}^{0}=\left(\begin{array}{c}
1 \\
\Pi_{0}^{*} \\
\Pi_{1}\left(1+\Pi_{0}^{*}\right) \\
\Pi_{1} \Pi_{2}\left(1+\Pi_{0}^{*}\right) \\
\Pi_{1} \Pi_{2} \Pi_{3}\left(1+\Pi_{0}^{*}\right) \\
\frac{S_{0}}{\mu}\left[f \gamma-\beta S_{0}\left(1+\Pi_{1}\right)\left(1+\Pi_{0}^{*}\right)\right] \\
\frac{1}{\mu}\left[(1-f) \gamma+f \gamma R_{10}-\beta R_{10}\left(\sigma_{1}+S_{0}\right)\left(1+\Pi_{1}\right)\left(1+\Pi_{0}^{*}\right)\right] \\
\frac{1}{\mu}\left[f \gamma R_{20}+\gamma \Pi^{* *}-\beta R_{20}\left(\sigma_{2}+S_{0}\right)\left(1+\Pi_{1}\right)\left(1+\Pi_{0}^{*}\right)\right]
\end{array}\right)
$$

and

$$
\mathbf{V}^{*}=\left(1,1, \frac{\gamma+\varphi_{1}+\mu}{\gamma+\varphi_{1}+\varphi_{2}+\mu}, 0,0,0,0,0\right)
$$

where

$$
\Pi^{* *}=\left(1+\Pi_{1}+\Pi_{1} \Pi_{2}+\Pi_{1} \Pi_{2} \Pi_{3}\right)\left(1+\Pi_{0}^{*}\right)-1 .
$$

Finally, denoting the $j$-th component of $V^{0}$ as $V^{0}(j), H^{\prime}$ is obtained as

$$
\left(\begin{array}{cccccccc}
\beta\left(V^{0}(6)+\sigma_{1} V^{0}(7)\right) & \beta\left(V^{0}(6)+\sigma_{1} V^{0}(7)\right) & \beta\left(V^{0}(6)+\sigma_{1} V^{0}(7)\right) & 0 & 0 & 0 & 0 & 0 \\
\sigma_{2} \beta V^{0}(8) & \sigma_{2} \beta V^{0}(8) & \sigma_{2} \beta V^{0}(8) & 0 & 0 & 0 & 0 & 0 \\
0 & 0 & 0 & 0 & 0 & 0 & 0 & 0 \\
0 & 0 & 0 & 0 & 0 & 0 & 0 & 0 \\
0 & 0 & 0 & 0 & 0 & 0 & 0 & 0 \\
-\beta V^{0}(6) & -\beta V^{0}(6) & -\beta V^{0}(6) & 0 & 0 & 0 & 0 & 0 \\
-\sigma_{1} \beta V^{0}(7) & -\sigma_{1} \beta V^{0}(7) & -\sigma_{1} \beta V^{0}(7) & 0 & 0 & 0 & 0 & 0 \\
-\sigma_{2} \beta V^{0}(8) & -\sigma_{2} \beta V^{0}(8) & -\sigma_{2} \beta V^{0}(8) & 0 & 0 & 0 & 0 & 0
\end{array}\right) .
$$

Therefore, we see from (4.5)-(4.7), that a backward bifurcation occurs for (4.1) at $\mathcal{R}_{0,3}=1$ if, and only if,

$$
V^{0}(6)+\sigma_{1} V^{0}(7)+\sigma_{2} V^{0}(8)>0
$$

or

$$
\begin{aligned}
& \left(1-\sigma_{1}\right) f \gamma S_{0}+\gamma\left(\sigma_{1}+\sigma_{2} \Pi^{* *}\right)> \\
& \quad \beta\left(1+\Pi_{1}\right)\left(1+\Pi_{0}^{*}\right)\left[S_{0}^{2}+\sigma_{1} R_{10}\left(\sigma_{1}+S_{0}\right)+\sigma_{2} R_{20}\left(\sigma_{2}+S_{0}\right)\right] .
\end{aligned}
$$

If $\varphi_{1}=0$ and $v_{2}=0$, (4.9) reduces to (3.4). It is clear that the likelihood of a backward bifurcation for (4.1) increases as $f$ increases. We shall first investigate the occurrence of backward bifurcations in absence of vaccination; then we will turn our interest to the impact of vaccination. Some parameter values are chosen from [2] and [8] and they are listed in Table 1.

\begin{tabular}{ccccccc}
\hline Parameters & $\beta$ & $\gamma$ & $\mu$ & $\varphi_{1}$ & $\varphi_{2}$ & $\varphi_{3}$ \\
Values & 9.045 & 6 & 0.03 & 8 & 3 & 0.03 \\
\hline
\end{tabular}

Table 1: Epidemiological parameters: $\beta, \gamma$, and $\mu$ taken from [2]; $\varphi_{1}, \varphi_{2}$ and $\varphi_{3}$ taken from [8].

CASE 1: $v_{2}=0$. 
Let $\sigma_{2}=0.75$. We know that $v_{1}$ and $\sigma_{1}$ are mutually determined by each other when the basic reproduction number $\mathcal{R}_{0,3}=1$. Fig. 3 shows a $f-\sigma_{1}$ region in which a backward bifurcation occurs at $\mathcal{R}_{0,3}=1$. On the other hand, for small $\sigma_{2}$ (e.g. $\sigma_{2}=0.5$ ), we find that the pairs $f-\sigma_{1}$ related by (4.3) do not satisfy (4.8) nor (4.9) and, therefore, no backward bifurcation occurs. This agrees with the observation in [22] that strong immunological protection against reinfection precludes the occurrence of a backward bifurcation. Our results also indicate that the extra behavioral protection among individuals with partial immunity can prevent the occurrence of backward bifurcation. However, it may also increase the likelihood of a backward bifurcation if individuals with partial immunity do not take any measures against transmission during sex (e.g. condom use).

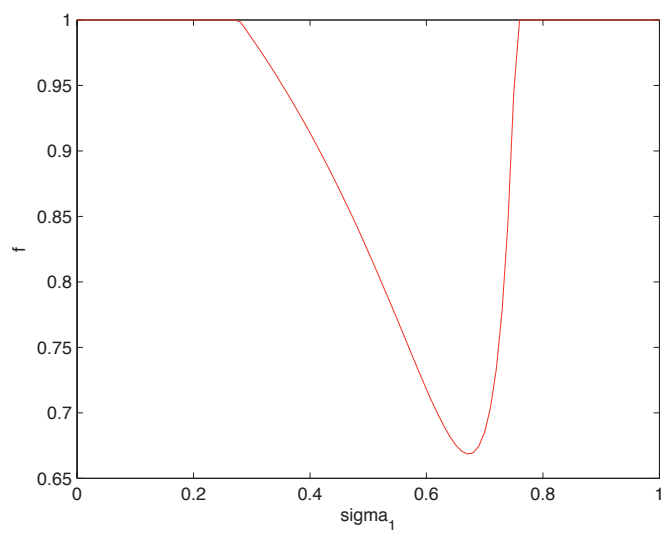

Figure 3: $f-\sigma_{1}$ diagram for a backward bifurcation. A backward bifurcation occurs in the region between the curve and $f=1$.

CASE 2. $v_{2}>0$ : Even though at present vaccination for syphilis is not yet used in clinics, it is still of interest to study the possible impact of vaccination on the dynamics of syphilis transmission in a population. Again, we use all the same parameters as in CASE 1. Fig. 4 shows that a backward bifurcation can be avoided with enough vaccination coverage, e.g. $v_{2}=0.02$ is almost good enough to accomplish this.

In order to eliminate syphilis in US, we need to ensure that the disease-free equilibrium is not only asymptotically stable but also a global attractor. In this section we derived a necessary and sufficient condition for a backward bifurcation when the basic reproduction number is 1 , which can be helpful in guiding health care policy.

\section{Discussion}

The interim targets of the Syphilis Elimination Effort in the US launched by the CDC are to reduce rate of primary and secondary cases [3]. However, weak immune response against reinfection after treatment in the primary stage, makes a backward bifurcation very likely, invalidating the sufficiency of the classical criterion $\mathcal{R}_{0}<1$ for elimination. This can be observed, for example, 


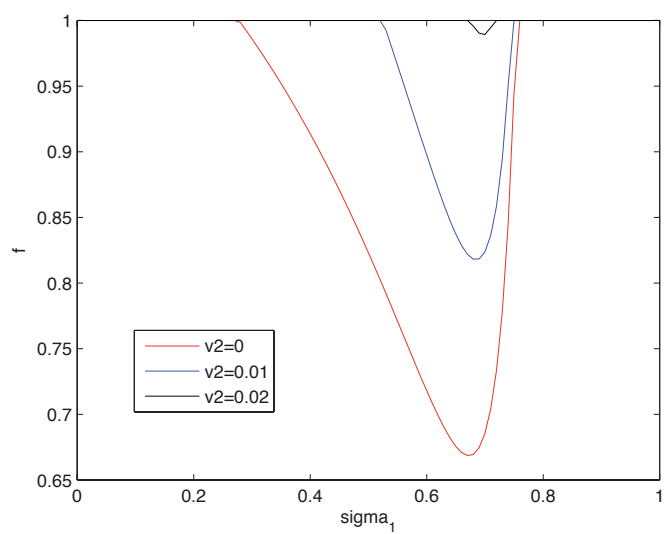

Figure 4: $f-\sigma_{1}$ diagram for different values of $v_{2}$. A backward bifurcation occurs in each case for values of $f$ and $\sigma_{1}$ within the region enclosed by the colored curve and $f=1$.

in a resurgence after a session of mass treatment [21]. Treatment in the primary stage increases the susceptible pool. If the control effort is not strong enough to avoid a backward bifurcation, a resurgence occurs following a dramatic decrease in prevalence when the basic reproduction number is decreased to just below 1 .

With experimental vaccines only one animal experiment thus far has shown complete immunity against reinfection. An effective vaccine of syphilis is still under research, but a vaccine conferring partial immunity is available. Behavioral protection plays a very important role in containing the spread of sexual transmitted diseases. Safe sexual practices such as proper use of condoms can lower the risk of infection. Our model indicates that the intensity of the epidemic (as measured by the reproduction number) will be lower after introducing vaccination, even with a vaccine that confers only partial immunity. Moreover, introducing vaccination can also lower the likelihood of a backward bifurcation.

However, behavioral protection may actually decrease among individuals recovered from primary infection and a backward bifurcation may arise. In particular, a backward bifurcation is very likely under the current strategies. We suggest that not only behavioral protection should be enhanced to eliminate syphilis in US, but effective vaccines should be applied as soon as possible.

We focused here on the impact of behavioral and biological protection on primary infection. Relapse of secondary syphilis $[1,18]$ usually appears one year after primary infection. Approximately one-fourth of syphilis infections develop into secondary relapse if not treated. However, it is very unlikely that such relapse will occur in modern society if treatment of syphilis infection is efficient and cheap. A similar analysis to the one we carried out here can be performed to focus on this specific stage of infection. 


\section{Acknowledgements}

R. Zhao would like to thank Dr. Sally Blower for valuable discussions.

\section{References}

[1] R. E. Baughn and D. M. Musher. Secondary syphilitic lesions. Clin. Microbiol. Rev., 18 (2005), 205-216.

[2] R. Breban, V. Supervie, J. T. Okano, R. Vardavas, and S. Blower. The transmission dynamics of syphilis and the CDC's elimination plan. Available from Nature Proceedings 〈http://dx.doi.org/0.1038/npre.2007.1373.1〉 (2007).

[3] R. Breban, V. Supervie, J. T. Okano, R. Vardavas, and S. Blower. Is there any evidence that syphilis epidemics cycle? Lancet Infect. Dis. 8 (2008), 577-581.

[4] Centers for Disease Control and Prevention. The National Plan to Eliminate Syphilis from the United States, 2006, http://www.cdc.gov/stopsyphilis/plan.htm.

[5] O. Diekmann, J. A. P. Heesterbeek, and J. A. J. Metz. On the definition and the computation of the basic reproduction ratio $R_{0}$ in models for infectionus diseases in heterogeneous populations. J. Math. Biol. 28 (1990), 365-382.

[6] L. Doherty, K. A. Fenton, J. Jones, T. C. Paine, S. P. Higgins, D. Williams, and A. Palfreeman. Syphilis: old problem, new strategy. BMJ, 325 (2002), 153-156.

[7] J. Dushoff, W. Huang, and C. Castillo-Chavez. Backwards bifurcations and catastrophe in simple models of fatal diseases. J. Math. Biol., 36 (1998), 227-248.

[8] G. P. Garnett, S. O. Aral, D. V. Hoyle, W. Cates, and R. M. Anderson. The natural history of syphilis: implications for the trasmission dynamics and control of infection. Sex. Transm. Dis., 24 (1997), 185-200.

[9] M. G. M. Gomes, A. O. Franco, M. C. Gomes, and G. F. Medley. The reinfection threshold promotes variability in tuberculosis epidemiology and vaccine efficacy. Proc. Biol. Sci., 271 (2004), 617-623.

[10] M. G. M. Gomes, A. Margheri, G. F. Medley, and C. Rebelo. Dynamical behaviour of epidemiological models with sub-optimal immunity and nonlinear incidence. J. Math. Biol., 51 (2005), 414-430.

[11] M. G. M. Gomes, L. J. White, and G. F. Medley. Infection, reinfection, and vaccination under suboptimal immune protection: epidemiological perspectives. Journal of Theoretical Biology, 228 (2004), 539-549. 
[12] D. Gökaydin, J. B. Oliveira-Martins, I. Gordo, and M. G. M. Gomes. The reinfection threshold regulates pathogen diversity: the case of influenza. J. R. Soc. Interface, 4 (2007), 137-142.

[13] N. C. Grassly, C. Fraser, and G. P. Garnett. Host immunity and synchronized epidemics of syphilis across the United States. Nature, 433 (2005), 417-421.

[14] K. P. Hadeler and P. Van den Driessche. Backward bifurcation in epidemic control. Mathematical Biosciences, 146 (1997), 15-35.

[15] A. K. Hurtig, A. Nicoll, C. Carne, T. Lissauer, N. Connor, J. P. Webster, and L. Ratcliffe. Syphilis in pregnant women and their children in the United Kingdom: results from national clinician reporting surveys 1994-7. BMJ, 317 (1998), 1617-1619.

[16] R. E. LaFond and S. A. Lukehart. Biological basis for syphilis. Clin. Microbiol. Rev., 19 (2006), 29-49.

[17] C. A. Morgan, S. A. Lukehart, and W. C. Van Voorhis. Protection against syphilis correlates with specificity of antibodies to the variable regions of Treponema pallidum repeat protein $K$. Infect. Immun. 71 (2003), 5605-5612.

[18] M. Myint, H. Bashiri, R. D. Harrington, and C. M. Marra. Relapse of secondary syphilis after Benzathine Penicillin G: molecular analysis. Sex. Transm. Dis., 31 (2004), 196-199.

[19] G. L. Oxman, K. Smolkowski, and J. Noell. Mathematical modeling of epidemic syphilis transmission: implications for syphilis control programs. Sex. Transm. Dis., 23 (1996), 3039.

[20] T. Parran. Syphilis: a public health problem. Science, 87 (1938), 147-152.

[21] B. Pourbohloul, M. L. Rekart, and R. C. Brunham. Impact of mass treatment on syphilis transmission: a mathematical modeling approach. Sex. Transm. Dis., 30 (2003), 297-305.

[22] T. C. Reluga and J. Medlock. Resistance mechanisms matter in SIR models. Math Biosci Eng., 4 (2007), 553-563.

[23] P. van den Driessche and J. Watmough. Reproduction numbers and sub-shreshold endemic equilibria for compartmental models of disease transmission. Mathematical Biosciences, 180 (2002), 29-48. 